\title{
IP Radio Resource Control System
}

\author{
John Vicente ${ }^{1,2}$ and Andrew T. Campbell ${ }^{1}$ \\ ${ }^{1}$ Center for Telecommunications Research, Columbia University \\ campbell@comet.columbia.edu \\ ${ }^{2}$ Intel Corporation \\ john.vicente@intel.com
}

\begin{abstract}
With the need for mobility and wireless communications now being motivated by the commercial sector, more attention must be given towards IP QOS in the wireless infrastructure, if existing or emerging services are to be commercialized over it. In this work, we investigate and propose a system approach to balance time-varying, space-varying (usage) wireless and mobile channel conditions against IP-based service differentiation and end-end service requirements. The proposed solution leverages network programmability [1], multiple timescales, feedback-based mechanisms across flows, mobile clients and a centralized RAN or LAN control point to configure QOS policies and coordinate state information between congestion control, bandwidth control and reliability mechanisms.
\end{abstract}

\section{Introduction}

The current convergence of wireless networks and the Internet is forging new developments in communications and networking services unlike any other time in the history of the telecommunications industry. In the area of quality of service (QOS), while the recent IETF efforts have made significant steps forward in QOS, the progress has been mainly driven from the perspective of a wired infrastructure. With the need for mobility and wireless communications now being motivated by the commercial sector, the same attention must be given towards QOS in the wireless infrastructure, if existing or emerging services are to be commercialized over it. However, the current Wireless Internet is a best-effort paradigm, and the traditional layered services fail to deliver reliable, beyond best-efforts services over an efficient, managed wireless infrastructure. Yet, these are the necessary requirements that information technology managers and service providers seek to ensure that their business customer needs will be met.

To achieve these objectives, several key philosophical barriers must be overcome or at least moderated. First, the end-end principles and in-network control must converge under the wireless, mobile paradigm. The applications ability to adapt to positive or negative wireless conditions, by either leveraging in-network services or 
binding alternative end-system services, will give it greater flexibility under a highly mobile and wireless environment. Second, a wireless evolution will require tighter layer integration and automation of application control and bandwidth management. The application's congestion control flexibility will depend on its ability to detect or respond on a much faster time-scale (e.g., to support fast handoff), thus requiring the network to cooperate with the flow's congestion control loop. Alternatively, the necessity to manage the wireless channel bandwidth will depend on the clocking rate and control mechanisms being used by the application to control the incoming rate of the flow. In this paper, we propose using a rate-based congestion control scheme as described in [2], as a more compatible scheme (vis-à-vis TCP windowing) with wireless traffic patterns and varying channel conditions. Third, we view existing layered or component services (e.g., TCP congestion control, 802.11 DCF, DiffServ) having overlapping or addressing uncoordinated functions including congestion management, service differentiation, bandwidth management and reliability. Fourth, this paper proposes that programmable networking [1] is needed to support this shift towards a Wireless Internet, thereby enabling new, dynamic services beyond the traditional barriers of current fixed services and QOS proposals. Finally, we propose that such a framework be legacy compatible in as much as is necessary not to burden the control system from efficient operation.

In what follows in Section 2, we present a systems approach to the problem of QOS, wireless resource control and flexible application adaptation in response to fluctuating conditions exhibited in the wireless environment. In Section 3, we present a programmable, open control system to support wireless IP resource control, along with a description of the components which make-up the proposed framework. Next in Section 4, we summarize additional work as follow-up to the current proposal.

\section{A Systems Approach}

In this work, we investigate and propose a system approach to balance wireless and mobile channel conditions against IP-based service differentiation and end-end service requirements. The underlying aspects of our proposal are summarized as follows:

- Tightly-couple resource management and control to achieve a stable, feedbackbased control system, combining both end-end and in-network control mechanisms,

- Leverage middleware (e.g., network APIs) services to "glue" components (legacy or otherwise) and policies in order to systemize the control framework,

- Maintain flow, local and global control separation, while dispatching or exposing specific control policies or state information between them,

- Discriminate control policy from time-varying channel conditions, fluctuating channel usage, or both. 
Figure 1 depicts a simple, conceptual model of our system goal to support flow adaptation in concert with local and global wireless channel resource management, and using both control and management signaling services to maintain (i.e., reliable, stable and performing) proper end-to-end flow delivery, optimal channel access and more efficient channel usage. By control, we are referring collectively to such services as QOS, congestion control, reliability and bandwidth control. By management, we are referring to monitoring mechanisms to support appropriate feedback to optimize control or policy-based decisions. In addition, we propose that control policies be based on conditions specific to the application flow, the local wireless device and the global channel. On a flow level, these conditions are at the scope of the application or connection transport level. The local device refers to the client mobile host, which may have time-varying conditions (e.g., fading, overlapped cells) imposed on its aggregate flows within its local vicinity, but may not necessarily reflect global conditions affecting all mobile hosts within the span of the wireless LAN or RAN. Alternatively, global conditions (e.g., channel traffic load) may affect the entire channel, and thus, all wireless devices within the same local area network.

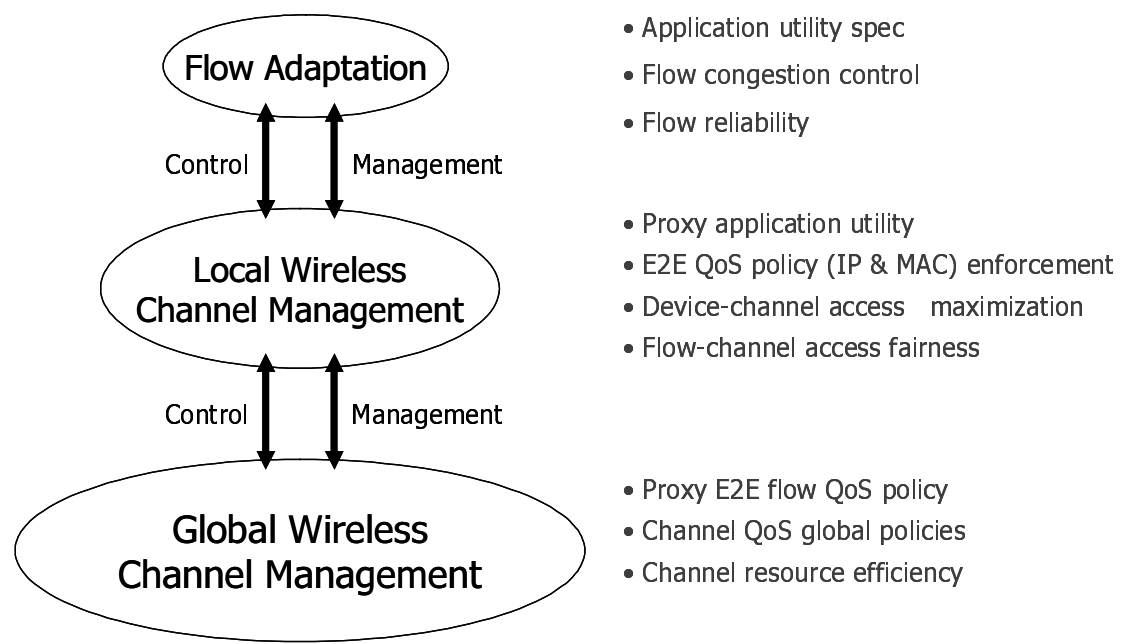

Fig. 1. Conceptual Model

Our approach does not suggest these three (i.e., state machines) as being independently managed or controlled. Instead, they must be cooperative in such a way, that each operates autonomously, however policies and states for their operation are exposed or exchanged for the intended stability and balance of the system operation. To achieve this, we consider an open network framework, where different components cooperate through exposed interfaces for binding purposes, dynamic configuration or state management. Furthermore, we can have greater control on the stability and efficiency of the system by enforcing policy controls at different timescales as needed to react, maintain or be proactive as warranted by the wireless device, the application flow or the wireless channel. 
To implement this system, we propose putting more cooperative bandwidth control into the IP stack and following along the same architectural lines of the UMTS Radio Resource Control component described in [3]. However, we recommend using the existing IP stacks with QOS extensions as defined by [4],[5],[6],[7] and providing more programmable integration across the layered boundaries. Furthermore, we argue for a rate-based congestion control scheme [2] as a more transport compatible scheme with wireless traffic patterns and varying channel conditions. We propose a middleware layer (e.g., CORBA) to automate cooperative control between a central wireless IP radio resource manager (RRM) handling RAN/WLAN global resource management and multiple, distributed RRM Agents at the mobile clients (and base stations). These agents would be working on behalf of the RRM to distribute global policies, while also managing local resource management and reacting to local channel conditions. The RRM Agent can be seen as mediation control point between the global channel (layer 2 and 3) policies, local (layer 2) channel policies and application flow adaptation at the transport-level (layer 4) and above. Figure 2 illustrates the proposed approach to IP-based wireless QOS and resource control.

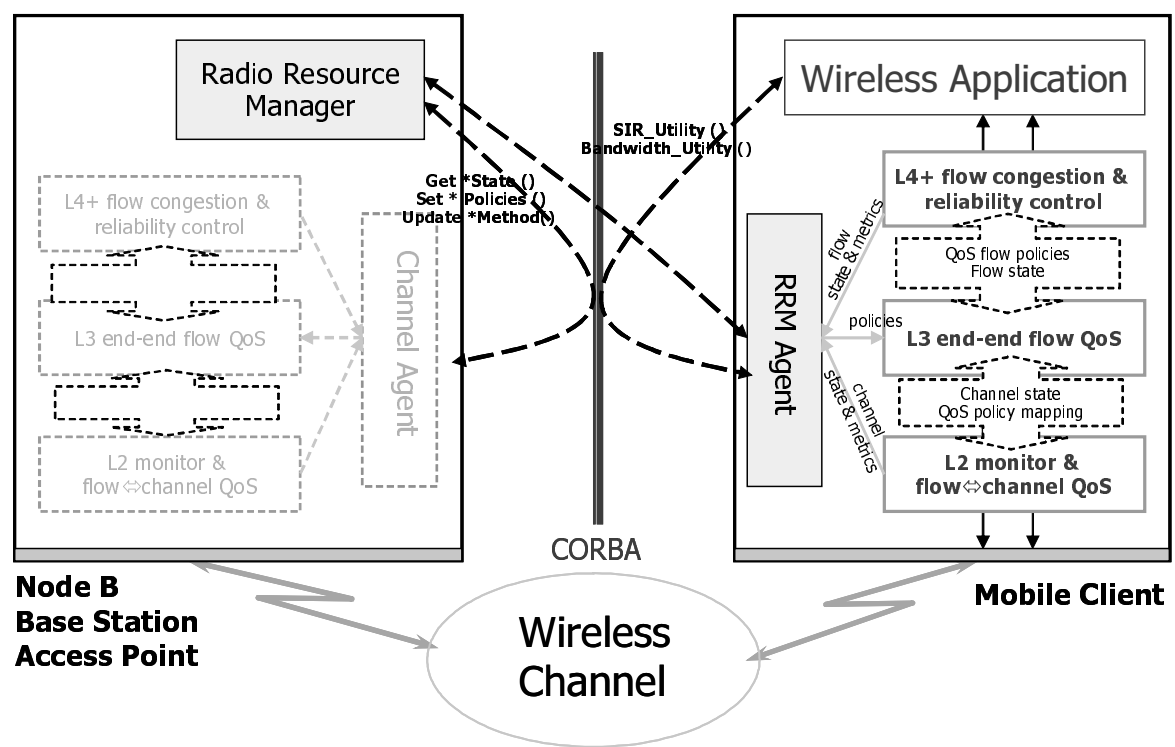

Fig. 2. IP Radio Resource Control Framework

\section{Wireless IP Resource Control System}

Figure 2 depicts a general model from which alternative wireless radio infrastructure can be supported via the Internet Protocol and supporting, enhanced IP-based network services. We do not propose an alternative network architecture (e.g., UMTS) nor new 
infrastructure elements (e.g., RNC), but instead, we envision a resource control framework, which is consistent with an evolving Wireless Internet. More specifically, we propose a framework that supports alternative radio link technologies, maintains the ubiquitous nature of IP, and is built on the layered transport and application services, which preserve the fundamental nature of the Internet's design. However, our framework supports the use of middleware services to enable a more dynamic, distributed and automated resource control strategy. Thus, the IP stack is exposed to support a more integrated QOS and resource control system, while preserving its layered boundaries. This open integration is needed for multiple reasons:

- Integrating the resource control hierarchy (i.e., end-to-end, global channel, local device, flow)

- Coordinating resource policies and states across multiple layers

- Binding alternative service mechanisms or algorithmic solutions

- Automating the resource control (provisioning and QOS maintenance) system

\subsection{System Components}

The motivation for our current work is based on [8], where we proposed a host-based network traffic control system for administering manual or automated QOS provisions based on algorithmically adjusting LAN resource and flow QOS policies. In [8], we use a programmable classifier and scheduler based on a traffic control API [9],[10] to dynamically, configure or enforce IP-based QOS policies (e.g., marking, shaping, metering, dropping, priority, etc) on-the-fly based on per flow and LAN usage feedback. In the current work, we believe the radio access network or wireless LAN environment is more suited for such a solution due to its highly dynamic nature. The wireless channel is a more autonomous system; not bearing the partitioned aspects of LAN switched or VLAN environments, which we perceived as an architectural

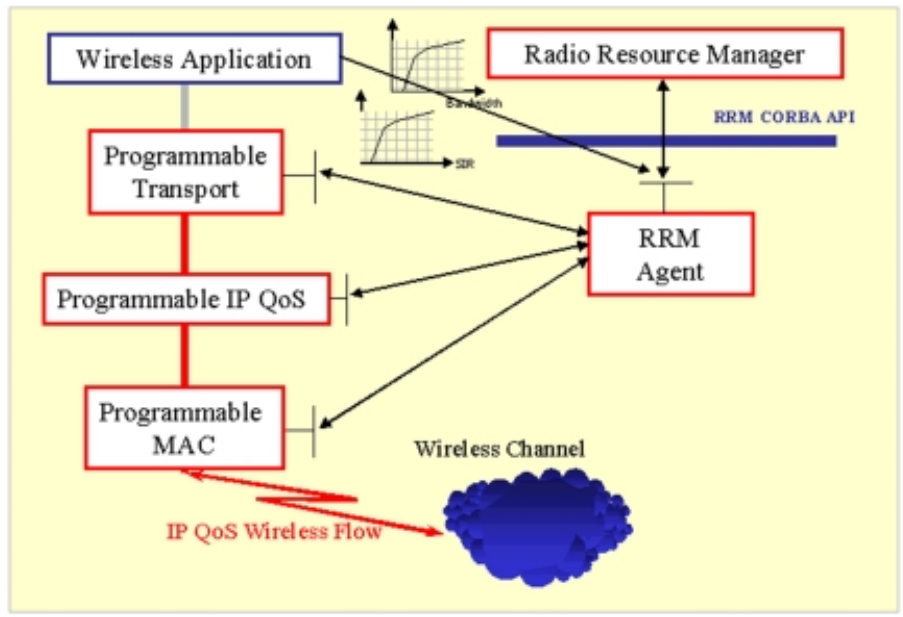

Fig. 3. Radio Resource Control Component Services 
limitation for the realization of the work in [8]. Thus, we extend the work in [8] by recognizing the need to feedback time-varying conditions and not just congestion or flow-level performance, and further, by distinguishing local feedback conditions from global conditions in making proper resource and QOS policy decisions. We also extend the previous work by enabling more dynamic features across multiple layers and providing support for utility-based, adaptation (see [11],[12],[13]). This latter requirement allows the wireless application to adapt to either local SINR or global channel congestion feedback. Figure 3 illustrates the resource control components in a distributed object structure.

Our work is not intended to address complete end-to-end SLA management, but assumes that the framework can be 'plugged-in' to a variety of policy-based or broker management systems, e.g., using COPS [14] for provisioning policy communications or CORBA for binding layered provisioning services. This contribution is geared towards an infrastructure-based wireless networking environment, however, we believe the proposed framework can be decentralized and peered to support an ad hoc wireless network environment as well. In either case, it is the spirit of this work to provide an 'auto-pilot' solution for automating resource control with dynamic provisioning in the face of a highly dynamic, mobile and time-varying wireless environment within a localized context. Finally, by maintaining the legacy aspects of the IP architecture, providing architectural separation from the underlying physical radio architecture, and using an API-based framework, it allows us to build upon the static nature of IP protocols and supporting layered services without introducing protocol or layer complexity to the same. Table 1 describes the system components supporting the proposed IP control framework. In what follows, we describe the design functionality of each of the components.

Table 1. System Components

\begin{tabular}{|l|l|l|l|}
\hline System Component & Timescale & \multicolumn{1}{|c|}{ Functions } & \multicolumn{1}{|c|}{ Programmable services } \\
\hline $\begin{array}{l}\text { Radio Resource } \\
\text { Manager }\end{array}$ & >seconds & $\begin{array}{l}\text { - Gateway E2E QoS services } \\
\text { - RAN resource control \& management } \\
\text { - RAN usage monitoring }\end{array}$ & $\begin{array}{l}\text { - RRM agent services } \\
\text { - Radio bandwidth monitoring }\end{array}$ \\
\hline RRM Agent & $\begin{array}{l}\sim \mathrm{msec} \text { - } \\
\text { seconds }\end{array}$ & $\begin{array}{l}\text { - Local device control \& management } \\
\text { - RRM Proxy }\end{array}$ & $\begin{array}{l}\text { - RRM proxy services to L2, L3, L4 } \\
\text { components } \\
\text { - Manage bandwidth \& SIR Utility }\end{array}$ \\
\hline $\begin{array}{l}\text { Programmable } \\
\text { Transport }\end{array}$ & $\sim 10 \mathrm{msecs}$ & $\begin{array}{l}\text { - Flow adaptation (congestion control, } \\
\text { loss \& delay packet monitoring) }\end{array}$ & $\begin{array}{l}\text { - Congestion control algorithms } \\
\text { - Flow policy \& state management }\end{array}$ \\
\hline Programmable IP QoS & $\sim 10 \mathrm{msecs}$ & $\begin{array}{l}\text { - E2E QoS policy enforcement } \\
\text { - Programmable QoS }\end{array}$ & $\begin{array}{l}\text { - Enforce bandwidth utility API } \\
\text { - E2E QoS policies } \\
\text { - Binding QoS algorithms/services } \\
\text { - Flow rate policing }\end{array}$ \\
\hline $\begin{array}{l}\text { Programmable } \\
\text { MAC }\end{array}$ & $\begin{array}{l}1 \mathrm{msec}- \\
100 \mathrm{msecs}\end{array}$ & $\begin{array}{l}\text { - Mapping of E2E QoS enforcement } \\
\text { - Local device adaptation } \\
\text { - Local SINR monitor }\end{array}$ & $\begin{array}{l}\text { - Enforce channel utility API } \\
\text { - QoS L3 } \rightarrow \text { L2 policy mapping } \\
\text { - Local SINR monitoring } \\
\text { - Binding QoS algorithms/services }\end{array}$ \\
\hline
\end{tabular}


Radio Resource Manager (RRM). The Radio Resource Manager is a central resource controller operating at 1 a radio network access point, base station or wireless LAN router. It can also act as a gateway to provision end-to-end QOS (e.g., from an ISP WAN) by allocating or managing local resources or mapping policies within the radio network. The RRM primarily oversees RAN resource control and management by negotiating wireless channel bandwidth requirements supporting its wireless clients while maximizing global channel bandwidth efficiency. RAN usage monitoring is critical to the automated resource control system by providing the feedback mechanisms at the highest level of RAN management. The RRM can employ alternative channel resource management schemes but uses distributed method invocations to dynamically adjust provisioning policies or program underlying layered component services resident at one or more wireless clients via the RRM Agent API.

RRM Agent. The RRM Agent is a distributed extension of the central RRM. The Agent is a mediation point balancing global channel resource policy, local channel policy, and flow-level adaptation. It communicates with client applications accepting application utility (utility-based SINR and bandwidth) functional specifications. Using RRM APIs, it communicates these utility specifications to the central RRM to support global channel optimization algorithms operating at the RRM. The Agent supports service programming (e.g., buffer management algorithm) or dynamic provisioning (e.g., marking, rate shaping policies), directly or via of the RRM. It accepts global policies delivered by the RRM and makes the necessary local enforcement decisions. Local channel resource control allows the link layer QOS service to adjust its scheduling and queuing mechanisms, temporarily preempting IP QOS policies in order to adjust access differentiation against time-varying conditions.

Inter-layer policy \& state synchronization. An important aspect of the RRM Agent is to coordinate policy and state between protocol stack layers to maintain constant synchronization or resynchronization induced either by global policy changes, local policy changes or flow adaptation. Such coordination may happen directly using header information (e.g., IP options), inter-layer header mapping (e.g., IP DSCP to 802.1p mapping) or via the RRM Agent through method invocation and parameter passing. The latter can be, for example, used for requesting/responding with monitoring state or QOS enforcement policies.

Programmable Transport. While the Internet Transport layer (TCP/UDP) has become one of the underpinnings in the Internet's end-to-end design philosophy, it has received much debate in terms of its flexibility to support real-time services, wireless infrastructure and mobility. Recent work (e.g., RTCP and [2]) have proposed alternative transport services necessary to match the requirements of emerging services. It is the general argument in this paper, that perhaps a rate-based congestion

1 Infrastructure mode. It is quite possible to decentralize the RRM function as a distributed system operating across nodes in an ad hoc network. 
mechanism [2] and flow measurement (e.g., latency, reliability) approach that supports faster congestion and drop type detection may be more appropriate for a wireless and mobile infrastructure. Furthermore, a programmable transport, where alternative transport layer control schemes can be employed or configured in real-time to provide greater flexibility to the multiple, varying conditions exhibited by the wireless channel. This is perhaps further complicated by a transport connection over several LAN/WAN networks consisting of both wireline and wireless underlying link transports. While we perceive that a programmable transport may create connection inconsistency across network nodes, a service mechanism, perhaps offered by the programmable transport may allow nodes to reconfigure the transport layer dynamically to synchronize transport services during connection setup.

Programmable IP QOS. In [8], we demonstrated that a flexible network layer QOS mechanism could allow automated provisioning and reconfiguration through threshold-triggered remote method invocation. Using centralized (multi-threaded) resource management algorithms, alternative IP flow QOS policies (e.g., token bucket parameters, packet marking, shaping, discard policies) could be enforced remotely over a common API exposed by client QOS agents.

In this paper, we propose a similar IP-based provisioning service at wireless devices, however we extend the previous work [8] to a greater context, allowing alternative IP QOS bindings and algorithmic choices enabling greater design and provisioning freedom to the IP radio QOS programmer or administrator. Moreover, through coordination with the RRM Agent acting as a local proxy, the IP QOS service can be (per application flow) configured through global policies algorithmically determined through bandwidth utility curves [11],[12] and managed by the centralized RRM. In this scenario, the RRM Agent obviates operational complexity between the various components, yet exposes necessary local methods and attributes allowing the layered components to coordinate with the Agent and communicate policies and state.

Programmable MAC. While noted progress has been made in the area of programmable wireless MACs [11] [12], [15], we adopt the notion of SINR utility curves [16] and apply this model towards differentiated access mechanisms supporting a programmable QOS link layer. Once again, through coordination with the RRM Agent, differentiated access can be (per application flow) dynamically (faster timescale) reconfigured through local policies algorithmically determined through SINR utility curves managed by the RRM Agent. In this scenario, the local policies may preempt the IP QOS global policies in order to manage time-varying or fairness issues caused by a fading or degraded local conditions on one or more flows. These policies may conflict with the end-end or global channel policies enforced at the higher layer. However, the higher layer QOS policies may become active once local channel conditions are improved. Figure 4 illustrates a current proposal [17] from the IEEE 802.11 [18] QOS Working Group on an Enhanced DCF supporting link layer QOS. We assume that IP network layer QOS policies are appropriately mapped down to MAC layer policies through pre-configured CoS/QOS mapping (e.g., 
DiffServ/802.1p header field mapping). QOS traffic classes are then assigned to appropriate EDCF queues along with associated per class contention window and QOS interframe space parameters. Thus, bandwidth, access, and latency differentiation is made possible at the local link level.

Finally, as in the network and transport layers, alternative MAC level QOS bindings or algorithmic choices should be allowed to be reprogrammed into the MAC layer. In the above example, each of the EDCF components may be reprogrammed or configured dynamically by the RRM Agent as needed.

- Prioritized output queues (queue[i])

- Legacy DCF finite state machine per queue (queue[i])

- CWmin differentiated per TC (CWmin[i]), controllable by EAP

- DIFS differentiated per TC (QIFS[i]), controllable by EAP

- Queue state machines count backoff slots in parallel

- Low-priority queues defer to higher-priority queues

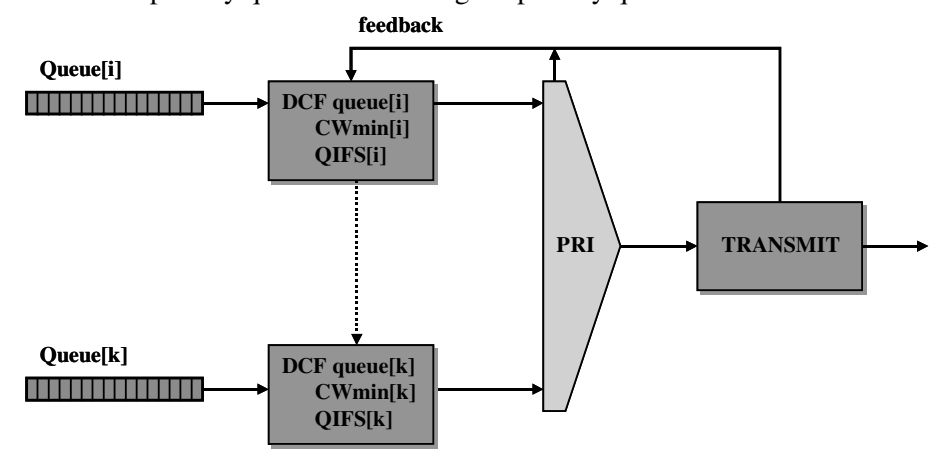

Fig. 4. Enhanced DCF, IEEE 802.11e IEEE 802.11-01/132r1 Proposal

\subsection{Flow Adaptation and Automated Resource Control}

The ideal Wireless Internet scenario has a user operating a real-time, multimedia videoconferencing session over a wide-area Wireless Internet connection. The wireless client is highly mobile within a local campus or dense city limits and is sharing a media rich presentation with multiple parties, dispersed in different connectivity scenarios (e.g., home, hotel, or corporate office). The user is experiencing a high degree of QOS supporting the multimedia session and presentation delivery, regardless of his movement or locality. While such a scenario seems far from current bandwidth and QOS paradigms, it is a motivation for a solution supporting wireless IP QOS and dynamic resource control. Multiple effects including time-varying channel conditions, local or remote congestion conditions, and end-to-end QOS requirements must be matched with a dynamic, automated resource control framework. Along with this, a flexible, adaptive application capable of offsetting the limitations of the network to bring to harmony reliability, latency and throughput degradations while hiding the user from the underlying complexity, degraded QOS, and mobility effects is 
also essential. To enable our solution, we promote the use of middleware enabled IP network services as necessary extension to the traditional IP architecture.

Resource Control \& Management Hierarchy. Fundamental to our approach is the need to integrate with an end-to-end QOS strategy. We support this at the IP layer through the Differentiated Services model presently under development by the IETF community; however, our framework must be complementary with other schemes (i.e., IntServ/RSVP) or new IP (or higher layer) QOS models as the Internet community creates them. Under a wide area Internet provisioning model, we utilize the role of the RRM to manage or proxy the end-to-end provisioning model against the resource requirements within the global wireless channel. The RRM can support an admission control scheme, such at defined in [19],[20] using policy-based management, or may integrate the requested provision in a more programmable fashion, where the provisioning request is invoked via a remote method, allowing the RRM, operating as a standalone admission control thread, to respond dynamically to the rapidly fluctuating conditions and policies of the environment. If the admission process is successful, the RRM must propagate new provisioning policies to specific RRM Agents, which must also invoke local methods to finalize admission and 'connect' the admitted session or flow. Otherwise, the RRM will decline admission and inform the outside party without the knowledge or participation of local RRM Agents. This separation is a necessary design feature of the distributed framework. In this paper, all provisioning aspects of the global wireless channel and the components within it are assumed autonomous and automated within the wireless resource controlled and managed domain.

Wireless channel policy control \& monitoring. As discussed in the previous section, the role of the RRM is to manage the end-to-end provisioning model against the resource requirements within the global wireless channel. Additionally, RRM monitors the channel for utilization 2 and keeps congestion within policy threshold limits pre-configured or adjusted throughout channel operation. There may exist multiple thresholds specific to the global channel utilization, specific QOS categories or alternative timescales, allowing the RRM to make granular policy decisions. This flexibility was also employed and demonstrated in [8]. By way of RRM APIs, the central RRM can accept application-specific bandwidth utility functions from RRM Agents on behalf of local applications and normalizes3 these functions to specific provisioning policies (e.g., DiffServ PHBs specs, RSVP QOS_Spec), which are provisioned locally or requested outside of the RRM provisioning realm (i.e., WLAN).

2 It is assumed that the RRM Agents will appropriately enforce and/or police flow QOS provisions, making it redundant that the RRM must do the same.

3 It is anticipated that such bandwidth utility functions may not be pervasive, however, they may be quite helpful to the RRM in its flexibility to accommodate adaptive applications. Alternatively, qualitative or quantitative specifications may be more likely, and thus, support for utility function normalization to simpler specification may be necessary. 
The scope of the bandwidth utility is global in nature and does not address adaptation to time-varying conditions related to local fading, overlapping cells, etc.

It is possible that the RRM may uniformly inform Agents of global channel usage conditions allowing the RRM Agents to tune policies at the transport (or application ${ }^{4}$ ) layer or local link layer components (e.g., MAC scheduler). This would support proactive control on a local device level.

Local policy control \& monitoring. The RRM Agent's scope is centered on its local environment. These Agents will reside on both wireless clients and wireless controller stations (e.g., base station or router). A critical function of the RRM Agent is to assess the time-varying conditions around the local device and manage this against the requirements of the running applications' flows and global channel effects. A SINR monitoring function is supported by the link layer to continuously fast monitor the time-varying conditions surrounding the wireless client as programmed by the RRM Agent. The fast timescale feedback, allows the Agent to adjust policies on a local level, allowing the client to compete more aggressively for the wireless channel. These updated policies can be specific to particular flows to ensure fairness, uniformly across all running flows on the client, or weighted (or prioritized) according to specific QOS specifications. Similar to the global bandwidth utility functions (discussed in 3.2.1.2), the RRM Agent exposes APIs allowing it to accept application-specific SINR utility functions [16] from local applications directly and translates these functions into specific, link layer provisioning policies. The scope of the SINR utility functions allow applications to adapt to local conditions at the device on a faster timescale.

State and policy information can be made available between layers by the RRM Agent to synchronize transport (per flow) congestion control and reliability states, network layer QOS policies and flow policing state, global usage and local SINR state. This capability is essential to the operation of the RRM Agent, allowing it dynamically control various layered components of the wireless client by leveraging multi-layer state or policies; extending its local intelligence (i.e., across application, transport, network, link layers) or influence on application adaptation.

Distributed State Machine Operation. In [8], we demonstrated that a flexible network layer QOS mechanism could allow dynamic provisioning reconfiguration either through manually (GUI-driven) invoked commands, or automatically through threshold triggered policy enforcement algorithms. These centralized and multithreaded (i.e., manual and pre-configured closed-loop threads) algorithms ran on a central, logical administrator handling the complete measurement-based control system, with the resulting policies dispatched to QOS agents operating at remote clients to enforce flow-level QOS policies. Our previous solution allowed the central administrator to operate in either a manual, user-driven provisioning mode or in an automated provisioning mode. However, under the wireless environment, we partition the control system across the resource control hierarchy. This is necessary to achieve

4 Informing the applications to consider employing alternative bandwidth savings or aggressive mechanisms or to dial down or dial up bandwidth utilities. 


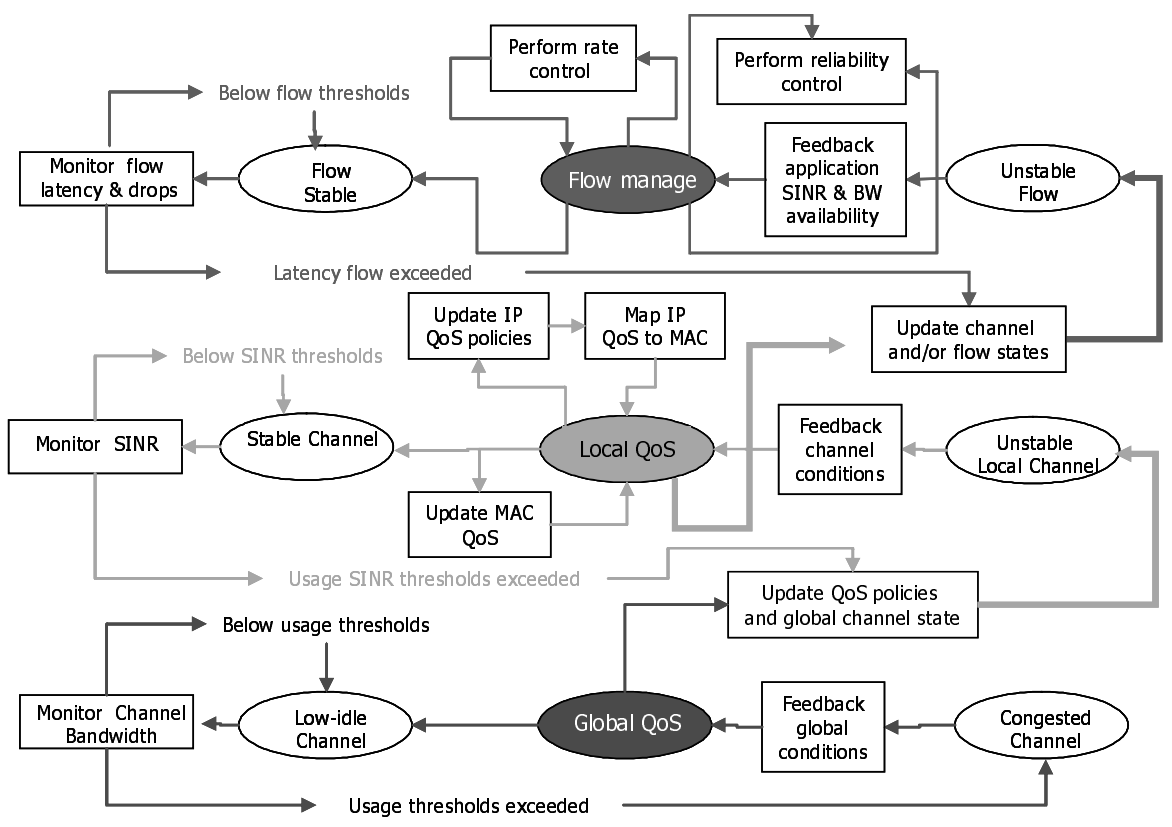

Fig. 5. Distributed State Machine

autonomous control on a global level, a local level and flow level; each of which is directed at a different set of objectives (e.g., global usage efficiency, local access maximization and fairness, and end-to-end flow control and adaptation), but overlapped on their influence on the wireless channel resource.

As illustrated in Figure 5, three autonomous levels of feedback-based control are supported in the distributed state machine. At each level, a stable and unstable state exists, while an operational state is centered between them to represent the control state. Also at each level, a monitoring procedure checks against stability thresholds to determine the possibility of instability and the need to enter into a control state, invoking alternative algorithms, which manage the particular level of concern. At the global and local level, policy changes will cause the state machine to enter into an unstable local channel state or unstable flow state, respectively. Multiple instances of the state machine procedure will run; one per wireless client and one for each of the flows running within the wireless environment. The flow procedure is essentially part of the normal transport process supporting both congestion and reliability control for each session flow. However, we expose it here as a necessary integration aspect of our framework. Also shown at the local and flow level is a procedure to update the application on specific bandwidth availabilities and SINR state, respectively. 


\section{Further Work}

While the proposed framework is still preliminary, we believe it is, nevertheless, quite feasible and appropriate for IP-based RANs or wireless LANs. However, a number open issues and areas require further investigation. The specific optimization algorithms, which can be run at the RRM and RRM Agent are clearly of open research. Inter-layer control interactions and their dynamics are also of open research and analysis. Since the various control and policy threads are happening on multiple timescales, we would need to explore their dynamics, both in terms of reaching appropriate stability and monitoring feedback synchronization. The programmability aspects also pose operational uncertainty when coupled with the traditional strict layering in the IP-based stacks. We intend to approach our investigations using simulation techniques.

In closing, with the convergence towards a Wireless Internet, it is our belief that automation of wireless resource and QOS control is necessary to match the highly dynamic nature of wireless and mobile environments. This paper has argued for an IPbased radio resource control system as the means to achieve this objective.

\section{References}

1. Campbell et al, "Mobiware Project", http://www.comet.columbia.edu/mobiware/.

2. Sinha P., Nandagopal, T., Venkitaraman N., Sivakumar R., Bharghavan V., "WTCP: A Reliable Transport Protocol for Wireless Wide-Area Networks", Wireless Networks, 1999

3. Holma, H., Toskala A., "WCDMA for UMTS: Radio Access for Third Generation Mobile Communications," John Wiley \& Sons, ISBN 0-471-72051-8

4. Bernet Y., Blake S., Binder J., Carlson M., Keshav S., Davies B., Ohlman B., Verma D., Wang Z, Weiss W., "A Framework for Differentiated Services," Internet-Draft draft-ietfdiffserv-framework-01.txt, work in progress, Feb. 1999.

5. Bernet Y., "The Complementary Roles of RSVP and Differentiated Services in the FullService QOS Network," IEEE Communications Magazine, Vol. 38, No. 2, pp. 154-162, Feb. 2000.

6. Braden R., Zhang L., Berson S., Herzog S., Jamin S., "Resource ReSerVation Protocol (RSVP)," Version 1 Functional Specification, RFC 2205, Sept. 1997.

7. Braden R., Clark D., Shenker S., "Integrated Services in the Internet Architecture: an Overview," RFC 1633, June 1994.

8. Vicente J. et al, "Host-based, Network Traffic Control System", Intel Technical Report (patent pending).

9. Ayyagari, A., Bernet Y., Moore T., "IEEE 802.11 Quality of Service -White Paper", IEEE 028 document, February, 2000.

10. Microsoft, "Quality of Service Technical Overview", http://www.microsoft.com/windows2000/library/howitworks/communications/trafficmgt/ qos.asp, Sept. 1999.

11. Bianchi G., Campbell A.T., Liao, R.R.F., "On Utility-Fair Adaptive Services in Wireless Networks", IEEE 1998. 
12. Barry M., Campbell A.T., Veres A., "Distributed Control Algorithms for Service Differentiation", Proc. IEEE INFOCOM'2001, Anchorage, Alaska, 2001.

13. Lee K., "Adaptive Network Support for Mobile Multimedia”, MOBICOM 95, Berkeley, CA, 1995.

14. Boyle J., Cohen R., Durham D., Herzog S., Rajan R., Sastry A., "The COPS (Common Open Policy Service) Protocol,” Internet Draft draft- ietf-rap-cops-07.txt. Aug. 1999.

15. Gomez J., Campbell, A.T., Morikawa, H. "A Systems Approach to Prediction, Compensation and Adaptation in Wireless Networks", WOWMOM 98, Dallas, Texas, 1998.

16. Liu X., Chong, E. K. P., Shroff, N.B., "Transmission Scheduling for Efficient Wireless Utilization", IEEE INFOCOM 2001.

17. Cervello G., Choi S., Qiao D., "Channel Model Proposal v2.0 for 802.11e MAC Simulations", IEEE document, April, 2001.

18. IEEE, "Wireless LAN Media Access Control (MAC) and Physical Layer (PHY) Specifications", IEEE Standard 802.11, January 1999.

19. Rajan R., Verma D., Kamat S., Felstaine E., Herzog S., "A Policy Framework for Integrated and Differentiated Services in the Internet," IEEE Network Magazine, Sept./Oct. 1999.

20. Yavatkar R., Hoffman D., Bernet Y., Baker F., Speer M., "SBM (Subnet Bandwidth Manager): A protocol for RSVP-based Admission Control over IEEE 802-style networks, draft-ietf-issll-is802-sbm-10.txt,” IETF Internet-Draft, January 2000.

21. Disabato C. M., Wireless LANs, The Burton Group, March 2001. 